\title{
Uncovering Hospitalists' Information Needs from Outside Healthcare Facilities in the Context of Health Information Exchange Using Association Rule Learning
}

D.A. Martinez'; E. Mora²; M. Gemmani2; J. Zayas-Castro³

1Johns Hopkins University, Emergency Medicine, Baltimore, MD, United States; ${ }^{2}$ Politecnico di Milano, Dipartimento di Ingegneria Gestionale, Milan, Italy; ${ }^{3}$ University of South Florida, Industrial and Management Systems Engineering, Tampa, FL, United States

\section{Keywords}

Health information exchange, medical record linkage, medical decision making, patient handoff, medical informatics applications

\section{Summary}

Background: Important barriers to health information exchange (HIE) adoption are clinical workflow disruptions and troubles with the system interface. Prior research suggests that HIE interfaces providing faster access to useful information may stimulate use and reduce barriers for adoption; however, little is known about informational needs of hospitalists.

Objective: To study the association between patient health problems and the type of information requested from outside healthcare providers by hospitalists of a tertiary care hospital.

Methods: We searched operational data associated with fax-based exchange of patient information (previous HIE implementation) between hospitalists of an internal medicine department in a large urban tertiary care hospital in Florida, and any other affiliated and unaffiliated healthcare provider. All hospitalizations from October 2011 to March 2014 were included in the search. Strong association rules between health problems and types of information requested during each hospitalization were discovered using Apriori algorithm, which were then validated by a team of hospitalists of the same department.

Results: Only 13.7\% (2089 out of 15230) of the hospitalizations generated at least one request of patient information to other providers. The transactional data showed 20 strong association rules between specific health problems and types of information exist. Among the 20 rules, for example, abdominal pain, chest pain, and anaemia patients are highly likely to have medical records and outside imaging results requested. Other health conditions, prone to have records requested, were lower urinary tract infection and back pain patients.

Conclusions: The presented list of strong co-occurrence of health problems and types of information requested by hospitalists from outside healthcare providers not only informs the implementation and design of HIE, but also helps to target future research on the impact of having access to outside information for specific patient cohorts. Our data-driven approach helps to reduce the typical biases of qualitative research. 


\section{Correspondence to:}

Diego A. Martinez, Ph.D.

Emergency Medicine

Johns Hopkins University

5801 Smith Avenue

Dvis Building Suite 220

Baltimore, MD 21209

Email: dmart101@jhmi.edu
Appl Clin Inform 2015; 6: 684-697

http://dx.doi.org/10.4338/ACI-2015-06-RA-0068

received: June 19, 2015

accepted in revised form: October 1, 2015

published: November 25, 2015

Citation: Martinez DA, Mora E, Gemmani M, ZayasCastro J. Uncovering hospitalists' information needs from outside healthcare facilities in the context of health information exchange using association rule learning. Appl Clin Inform 2015; 6: 684-697 http://dx.doi.org/10.4338/ACl-2015-06-RA-0068 


\section{Introduction}

In the United States, people suffering from chronic health conditions constitute $49.8 \%$ of the adult population [1], and they consume $84 \%$ of the health care expenditures [2]. For people to achieve a safe, effective, and efficient health care, a coordinated effort is often required among unaffiliated providers. Lack of care coordination may lead to medication errors, avoidable hospital readmissions, duplicated testing, and delays in understanding the patient condition [3-10]. Since 2009, to support improvements in care coordination, the federal government has been stimulating the adoption and use of health information exchange (HIE). However, recent studies report HIE adoption across hospitals is still low $[11,12]$. As noted in the systematic review by Rudin and colleagues, one of the important barriers to HIE adoption are clinical workflow disruptions and troubles with the system interface [13]. Several authors claim better-designed interfaces for HIE systems would stimulate its usage since clinicians will have quicker access to useful patient information [14-16].

To improve HIE systems, it is imperative to understand physician information needs from outside health care facilities. Healthcare providers are increasingly constrained by the time they have to diagnose and treat patients, while trying to both follow evidence-based recommendations and consider the unique needs, characteristics, and preferences of the patients [17-22]. Given that the voluntary usage of additional information sources, such as HIE, can be discouraged by time constraints [23], there is a need to make the information displayed on HIE systems more valuable than the opportunity costs. For instance, screen redesign, single sign-on, enhanced record searches, or eliciting user needs could all be means to address the need. Additionally, the expected benefits of HIE might be fruitless if clinicians do not have access to a system that takes into account users' unique needs, cognitive tasks, and workflow processes [24]. However, there is no clear understanding and agreement of what data elements are needed from outside health care facilities to assist physicians in their decision-making [25]. Therefore, the information needs of the physicians are needed to inform the design and deployment of the HIE and health IT policy. Most of the published studies on physicians' information needs have focused on the communication between hospitalbased (i.e. hospitalists) and primary care physicians [26]. However, in the context of HIE, the information sharing will include a bigger spectrum of healthcare providers. The communication between hospitalists and primary care providers has particular perspectives that may influence information needs and resource preferences.

Additionally, the collection of meaningful data on information needs may be problematic. Beyond the usual drawbacks of surveys and interviews, physician self-assessments of informationseeking behavior can be unreliable. For example, physicians may be unaware of their needs at the time of applying the self-assessment instrument. The information channels they use and their methods of using them, which are influenced by study habits adopted as early as medical school or college, may not provide the most efficient, accurate, and comprehensive information necessary for medical decision-making [27]. Many physicians are unaware of, or uncomfortable with, ever-evolving sources of information. In previous years, investigations have used questionnaires [28-32] and interviews [33-36] to shed light on physician's sources of information and how these influence workflow. Unfortunately, limited conclusions can be drawn from these data due to limitations in the internal validity and generalizability. In many of the investigations, for example, less than $50 \%$ of the sample population participated in the study.

This article reports the results of a study to document hospitalists' information needs in a large urban tertiary care hospital in Florida with no HIE functionality, and in planning stages for implementation. Our objective was to uncover associations between the health problems of the patient and the type of clinical information requested from outside health care facilities. An attempt was made to reduce selection and recall biases by mining a large number of data transactions from October 2011 to March 2014 of all hospitalists and residents working in the internal medicine department. Since other researchers have successfully used association rule learning (ARL) algorithms to analyze healthcare data [37-39], we implemented the Apriori algorithm to discover strong associations between the patients' health problems and the clinical information requested. The outcome of our investigation will help HIE developers and implementers recognize commonly requested clinical information from outside health care facilities by specific health problems, and thereby prioritize information display. 


\section{Methods}

The transactional data used in this study were collected from the Internal Medicine Department of Tampa General Hospital (TGH) in Tampa, Florida. TGH is a 1018-bed tertiary care hospital serving over four million people from 23 counties in West Central Florida with no HIE functionality, and in planning stages for implementation. During the study timeframe, there was no functional HIE in the region where TGH is located, and thereby most of the health information transactions between healthcare providers were performed via fax and telephone. A list of disease-information association rules was mined from transactional data using the Apriori algorithm, and validated by senior internists working at the same department. Transactional data includes all types of clinical information requested from outside healthcare providers during a patient hospitalization (denoted as outside information, OI) via fax and telephone, which was then scanned into the patient's electronic medical record. Our approach comprised four major phases: data collection and pre-processing, association rule building, post-processing and association rule selection, and clinical expert validation.

\subsection{Data Collection and Pre-processing}

Our dataset included all hospitalizations from October 2011 to March 2014 with at least one request for OI. The dataset was constructed with the list of health problems, and the list of OI requested in each hospitalization. The list of health problems corresponds to the discharge problem list, which are directly recorded by physicians during the patient hospitalization. We also collected demographic and clinical factors associated with each hospitalization. Independently, to assure consistency, three co-authors detected and corrected inaccurate health problem terms in the dataset. Any discrepancies between the co-authors were discussed and resolved by consensus, and uncertainty was referred to the fourth co-author.

\subsection{Association Rules Building}

We used ARL to discover strong associations between the health problems (antecedent) and OI requested (consequent). Since previous investigations found HIE useful only in particular cases [40], we hypothesize that a strong association between a health problem and an OI type indicates important information need. Association rules are antecedents implying consequences of the form $X \rightarrow Y$, in our study, health problems implying OI requests. The association $X \rightarrow Y$ measures how likely the event $Y$ is, given $X$. We measured the quality of an association rule in terms of support and confidence, and the quality of an association rule in terms of lift. Support corresponds to the statistical significance of a rule given by the proportion of transactions in the dataset containing a given set of health problems and OI types. A high support denotes a high popularity for the given set of health problems and OI types. Confidence is a measure of a rule's strength and is calculated as the conditional probability of the consequent given the antecedent, which is understood as the probability that a health problem occurs if it is known that a particular OI type was requested. Lift denotes the strength of the rule over the random co-occurrence of the antecedents and the consequent. Particularly, a lift greater than one implies the association between the set of health problems and the set of OI types is more significant than if the two sets were independent. In our context, an association rule with a lift value of two means that a physician who serves a patient with disease $X$ is twice more likely to request outside information type $Y$ than the general physician, and similarly, the physician who request $Y$ is twice as likely to being serving a patient type $X$, since lift is a symmetric measure. The stronger the association is - the larger the lift. In epidemiological terms, support and confidence are related to the terms of prevalence and positive predictive value, respectively.

The association rules were mined using Apriori algorithm [41], which was executed in $\mathrm{R}$ using the Arules package [42]. Apriori calculates a set of strong rules given an arbitrarily selected minimum value for support and confidence. The strategy behind Apriori is to decompose the task of finding strong rules into two major subtasks; the frequent itemset generation and the rule generation. Frequent itemset generation finds those itemsets satisfying an arbitrarily selected minimum support value. On the other hand, rule generation extracts all the high-confidence rules from the previously generated frequent itemsets. These extracted rules are denoted as strong rules. Apriori 
algorithm assumes items within an itemset to be independent, and thereby it may disregard hidden interrelationships among items. This is important when dealing with many real-world applications since the data under study are usually far from being perfect. For example, a distributed information environment with data being collected from different sources with imprecise and vague documentation methods. In our study, we assume that the dataset under study is precise and contain no ambiguity. We support this assumption in the fact that all data collected for this study were documented by highly trained individuals in a single EMR system. More precisely, hospitalists document the health problems during a hospitalization and coders from the hospital electronic medical records department document the OI types received from outside healthcare providers.

\subsection{Post-processing and Association Rule Selection}

Once the set of strong rules was generated, we selected those in which both of the following conditions were satisfied: at least one health problem was present in the antecedent, and at least one OI type was present in the consequent. We denote these extracted rules as strong and potentially meaningful rules. Additionally, a chi-square test was utilized to determine the statistical significance of each association rule, where the rule-corresponding two-by-two table is given by the cells $X \cap Y, X^{c} \cap Y$, $X \cap Y^{c}$ and $X^{c} \cap Y^{c}$ where $c$ refers to the complement of a given itemset. To facilitate calculations, we used the results of [43] to derive the chi-square value of each rule in terms of its support, confidence, and lift, and of the total number of data instances $n$. A p-value providing an upper bound on the type I error (i.e. the risk of discovering a rule that is actually false) of each rule is then computed from the chi-square value by consulting the chi-square distribution with one degree of freedom. Due to the high risk of type I error inherent to ARL algorithms, we adjusted the p-values to control for false discovery using an improved Bonferroni-type procedure: the Benjamini-Hochberg correction method [44]. This method allows us to control type I error during the identification of statistically significant rules in our exploratory study. Another approach to evaluating the statistical significance of association rules is to test tentative rules on a validation dataset. However, this approach is problematic to use in exploratory studies, as in our context, due to the limited data availability. In our study, we consider those rules for which the chi-square values lead to a corrected statistical significance level or type I error of 0.10 or lower to be statistically significant.

\subsection{Clinical Expert Validation}

We validated the set of strong and potentially meaningful rules with three internists from the TGH Internal Medicine Department. To assure consistency, the three internists independently assessed the set of rules generated by our research team. By consensus, any discrepancies between the internists were discussed and resolved. These validated rules are denoted as our final set of association rules.

\section{Results}

\subsection{Population and Dataset}

Only $13.7 \%$ (2089 out of 15230) of the hospitalizations in the internal medicine department generated at least one request for OI. As shown in Table 1, 50.7\% of the patients were female, with $93.2 \%$ English speakers followed by $4.5 \%$ Spanish speakers. Although $91.9 \%$ of the patients were admitted through the emergency department, most of them $(59.1 \%)$ had a primary care provider at the time of their admission. The mean age was 53.5 years old, and the mean length of stay was 6.7 days. Hospitalists from the internal medicine department under study do no routinely collect OI, and if they do, the patient or their relatives have to authorize the released of patient information from outside healthcare facilities. As noted in - Table 2, 75\% of the requests for OI are made within 22 hours from patient admission and only $10 \%$ of the requests are made within one hour. Based on this data, the OI requests were not part of a routine during patient admission, and they seem to play an important role, perhaps, when the clinical picture of the patient becomes less clear than initially appeared. 
The most common health problems and OI requested in the 2089 hospitalizations under study are presented in Table 3. The majority of the requests for OI were from rather non-specific health problems such as chest pain, $18.5 \%$, abdominal pain, $15.1 \%$, and dyspnoea, $9.9 \%$. This pattern is aligned with the patient population and clinical setting under study. On the other hand, the most frequent OI requested were outside medical records with $77.9 \%$, followed by laboratory test results with $18.5 \%$ and imaging results with $18.2 \%$. Important to note is that the frequency analysis presented in $>$ Table 3 may result in overlap between the different classes of health problems and outside information types.

\subsection{Association Rules}

The final set of association rules is presented in Table 4 . We fixed the minimum support at $2 \%$, minimum confidence at $75 \%$, lift values greater than one, and the association rules had to have at least one health problem in the antecedent and one OI type in the consequent. Clinically relevant rules are presented in $>$ Table 4 . A total of 20 association rules were found to be clinically relevant, of which the two with the lowest p-values (rules 3 and 16 in Table 4) do not satisfy. By the Benjamini-Hochberg correction method, we concluded that since $0.01=(2 / 20) 0.1$, these two results are not statistically significant at the corrected level $\mathrm{P}<0.1$. All of the rules were determined by chi-square analysis and Benjamini-Hochberg correction not to be significant. Although our conservative approach resulted in no statistically sound association rules, there seems to be a trend between health problems and OI types for specific patient cohorts. For example, in terms of support, the stronger association rules found are \{abdominal pain $\rightarrow$ outside medical records\} and anaemia $\rightarrow$ outside medical records\}. That is, outside medical records are frequently requested for abdominal pain and anaemia patients with a support of $12 \%$ and $10 \%$, respectively. When requesting OI for abdominal pain patients, there is an $83 \%$ confidence of requesting outside medical records. Similarly for anaemia patients, there is an $80 \%$ confidence of requesting outside medical records. The Internal Medicine Department usually serves people carrying several chronic conditions as comorbidities of an acute condition. Hence, most of the requests for outside medical records were for chronically ill patients. Despite this fact, the collected data show acute cases such as lower urinary tract infections typically trigger requests for outside medical records as well. For this particular patient cohort, there is an $86 \%$ chance of requesting outside medical records. Other acute conditions found among the 20 strong association rules were patients with abdominal pain, chest pain, nausea, and vomiting.

\section{Discussion}

We sought to uncover the relationship between the patients' health problems and the information needed from outside health care facilities in a large academic medical center. ARL was used to mine two and a half years of transactional data from the hospital EMR previous HIE implementation. Although previous investigations have made valuable contributions to the knowledge base on informational needs of physicians and patterns of use of HIE systems [45, 46], most of them focus solely on hospital and primary care provider communication. We construct on these investigations considering the entire spectrum from which a hospital physician (i.e. hospitalist) may request patient records. With an increased number of handoffs between providers [47], due to the shift towards hospital medicine, studying informational needs of hospitalists becomes essential for improving HIE functionality, and thereby reducing barriers to adoption. We have also identified an important gap in the literature - most of the HIEs are built and implemented without first performing a user needs assessment. We believe HIE will be more successful if it is evaluated before, during and after implementation. To the best of our knowledge, there is no previous study serving as both needs assessment and baseline of informational needs prior to HIE implementation. Important to note is that hospitalists working in the department under study identified specific situations where they know outside information exists, but they do not request for records. For example, physician assumes the OI request process takes too long or the patient does not know where to request outside information from. These situations are amenable to HIE; therefore, physician OI request behavior may change after HIE implementation. We plan on capturing these variations in a future study. 
Previous investigations suggest users have determined HIE is useful in some, but not all cases [40]. Our results indicate those patients hospitalized with chest pain were the target of outside information requests to obtain EKG results and other imaging test results. Other patient cohorts that were a common target of outside information requests were urinary tract infection patients and back pain patients. Indeed, Bailey and colleagues found HIE usage was associated with $64 \%$ lower odds of repeated imaging testing for back pain patients [6]. These findings can be translated into HIE design recommendations; for example, HIE systems should provide one-click access to imaging, echocardiograms, bacterial cultures, cardiac catheterizations and CT scans allocated in other healthcare facilities for those patients with acute cardiac issues, urinary tract infection and back pain. Not only did our results indicate which patient populations are more prone to have outside records requested, but they indicated where future HIE research should focus to elucidate the value of information exchange among providers. Still, work lies ahead in elucidating whether or not streamlined access to outside information improves medical decision-making for other patient populations, and hence lower health care costs and improve patient outcomes. Future research should focus on determining the effects of having quick access to outside information in those patient cohorts previously unexplored; for example, urinary tract infection patients. Additionally, we would like to point out that few hospital transfers and physician referrals were included in our study. Since previous research found that incomplete patient records during transfers may lead to costly duplicated testing [48], future investigations should focus on the role of HIE during the admission of transferred patients.

A crucial step in improving information exchange between inpatient and other settings of care is the discharge summary [49-51]. Although The Joint Commission on Accreditation of Healthcare Organizations requires a discharge summary for every patient, usually, they do not provide timely and sufficient information for appropriate care transitions [52-54]. Kripalani and colleagues, in their 2007 systematic review of deficits in communication and information transfer between hospitalists and primary care physicians, infer that new health information technology and standardized methods of information exchange bears particular promise to improve care coordination [26, 45]. Computer-generated summaries offer a quick way to present and highlight key elements of the hospitalization, and they are ready for delivery sooner than traditional summaries [55]. However, information needs and collection habits are not generic but instead vary among different types of physicians. Previous investigations found information needs and expectations of computers are influenced by specialty and practice setting $[28,33,56,57]$. Future research must determine differences between informational needs due to a variety of factors that include the young physician's lack of experience with fundamental clinical principles and the senior physician's lack of experience with information technology.

We found few other studies analyzing informational needs in the context of information exchange among healthcare organizations. Two studies, focused on the emergency department (ED) and outpatient care settings, found most OI users accessed patient summary data displayed by default in the HIE system followed by detailed laboratory and radiology information, which is consistent to what we found $[58,59]$. We contribute to this body of research by focusing on the inpatient care setting and hospitalists, who are key actors in coordinating the care of the patient within and outside the hospital. Ozkaynak and Brennan, during direct observation of ED workflows, found clinicians were more likely to request OI for admissions of chronic pain patients [60], which is consistent with our findings as well. However, during follow-up interviews, they found ED clinicians requested OI to identify drug seekers, which may not be the same motivation of hospitalists. Further research should explore hospitalists' perceptions on the value of OI to support medical decisionmaking.

There are important limitations to our work. First, we do not know if information-seeking efforts of hospitalists were successful. The collected transactional data have no information on whether or not the user located the desired information. Important to note is that when OI arrives, hospitalists must wait for the OI to be scanned into the hospital EMR to have access to the information. Therefore, the chances of having users receiving but not scanning the OI into the EMR are low. Second, our study was restricted to a single hospital and thus a single EMR. However, most of the features of the in-use EMR were the same as the majority of hospitals across the nation. Third, the results of this work have limited generalizability in terms of the setting of care. Information users from other 
settings of care, even within the same hospital, may have different information needs. Yet, in the presence of data, our methodological approach can be reproduced to elucidate information needs in other clinical settings. Fourth, the usage of direct communication to verbally request OI (i.e. telephone call to the outside healthcare provider), which is then directly documented by the clinician in the patient's medical record were not included in this study. Finally, we did not address potential confounding due to region characteristics (e.g. the number of unaffiliated outside healthcare providers and their electronic medical record adoption rates).

\section{Conclusion}

We proposed a new approach to studying informational needs of clinicians in the context of HIE. In particular, we uncovered the relationship between health problems and the most critical information requested, from outside health care facilities, in an internal medicine department of a tertiary care hospital. After data preparation, a set of disease-information association rules was built and then validated by clinical experts. This knowledge should inform the design and implementation of HIE in similar clinical settings, and in the presence of data, our approach can be used in other clinical settings as well. Our study contributes to filling the existing gap in knowing and understanding the clinical information needs in the context of new health information technology. With better knowledge of clinical information needs, it will become possible to conduct prospective studies of the clinical benefit of providing doctors with decision support tools that meet their outside information needs. Evidence can then be collected on whether improved access to outside information will result in more efficient or effective clinical decision-making or improved patient health outcomes. The effectiveness of health information exchange can thereby obtain its most eloquent validation.

\section{CLINICAL RELEVANCE STATEMENT}

Health information exchange is expected to facilitate a better delivery of care to patients. This study assists that goal by uncovering the most commonly requested clinical information from outside health care facilities by specific health problems. In the hands of HIE developers and implementers, our framework may facilitate screen redesign and enhanced record searching, and thereby reduce clinical workflow disruptions and troubles with the system interface.

\section{CONFLICT OF INTEREST}

The authors declare that they have no conflicts of interest in this study.

\section{HUMAN SUBJECTS PROTECTIONS}

This study was performed in compliance with the World Medical Association Declaration of Helsinki on Ethical Principles for Medical Research Involving Human Subjects, and was reviewed by the Tampa General Hospital Office of Clinical Research and the University of South Florida (IRB \#Pro00014574).

\section{ACKNOWLEDGEMENTS}

We thank Alexandra Strauss, Candice Mateja, and Stephanie Taylor for their valuable contributions in our study. We also thank Andres Garcia-Arce for his contributions during early stages of this project. Finally, we would also like to show our gratitude to the four anonymous reviewers for their comments that greatly improved our manuscript. 


\begin{tabular}{|c|c|}
\hline$N=2089$ & No. $(\%)$ \\
\hline Female & $1059(50.7)$ \\
\hline \multicolumn{2}{|l|}{ Language preference } \\
\hline English & 1948 (93.2) \\
\hline Spanish & $94(4.5)$ \\
\hline Unknown/Other & $47(2.3)$ \\
\hline \multicolumn{2}{|l|}{ Marital status } \\
\hline Single & $1361(65.1)$ \\
\hline Married & $650(31.2)$ \\
\hline Unknown/Other & $78(3.7)$ \\
\hline Have a primary care provider & $1235(59.1)$ \\
\hline \multicolumn{2}{|l|}{ Payer class } \\
\hline Commercial & $627(30)$ \\
\hline Medicare & $817(39.1)$ \\
\hline Medicaid & $465(22.2)$ \\
\hline $\mathrm{HCHCP}$ & $137(6.6)$ \\
\hline Other & $45(2.1)$ \\
\hline \multicolumn{2}{|l|}{ Admission source } \\
\hline Emergency room & 1919 (91.9) \\
\hline Physician-referral & $84(4)$ \\
\hline Outside hospital & $84(4)$ \\
\hline Other & $2(0.1)$ \\
\hline \multicolumn{2}{|l|}{ Mean (SD) } \\
\hline Age & $53.5(17.3)$ \\
\hline Length of stay & $6.7(10.0)$ \\
\hline
\end{tabular}

Table 1 Demographic and clinical factors of hospitalizations, with at least one request for clinical information from outside healthcare providers, in the Internal Medicine Department of the Tampa General Hospital.

HCHCP: Hillsborough Country Health Care Plan

\begin{tabular}{|l|l|l|}
\hline Quantile & Duration in minutes & Duration in hours \\
\hline $100 \%$ Max & 51894 & 865 \\
\hline $99 \%$ & 18456 & 308 \\
\hline $95 \%$ & 6072 & 101 \\
\hline $90 \%$ & 3534 & 59 \\
\hline $75 \%$ Q3 & 1309 & 22 \\
\hline $50 \%$ Median & 575 & 10 \\
\hline $25 \%$ Q1 & 224 & 4 \\
\hline $10 \%$ & 49 & 1 \\
\hline $5 \%$ & 23 & 0 \\
\hline $1 \%$ & 0 & 0 \\
\hline $0 \%$ Min & 0 & 0 \\
\hline
\end{tabular}

Table 2 Analysis of duration from patient admission to when the request for Ol was made by a hospitalist in the Internal Medicine Department of Tampa General Hospital. 


\begin{tabular}{|c|c|}
\hline Health Problems & Number of hospitalizations (\%) \\
\hline Chest pain & $387(18.5)$ \\
\hline Abdominal pain & $315(15.1)$ \\
\hline Anemia & $261(12.5)$ \\
\hline Dyspnoea & $206(9.9)$ \\
\hline Hypertension & $199(9.5)$ \\
\hline Diabetes mellitus & $195(9.3)$ \\
\hline Leukocytosis & $182(8.7)$ \\
\hline Renal Failure & $177(8.5)$ \\
\hline Vomiting & $152(7.3)$ \\
\hline Nausea & $150(7.2)$ \\
\hline Altered mental status & $133(6.4)$ \\
\hline Fever & $122(5.8)$ \\
\hline Cancer & $109(5.2)$ \\
\hline Tachycardia & $107(5.1)$ \\
\hline Hypotension & $100(4.8)$ \\
\hline Lower urinary tract infection & $97(4.6)$ \\
\hline Hypokalaemia & $96(4.6)$ \\
\hline Hyponatraemia & $92(4.4)$ \\
\hline Back pain & $88(4.2)$ \\
\hline Syncope & $88(4.2)$ \\
\hline Coronary artery disease & $84(4.0)$ \\
\hline Pneumonia & $81(3.9)$ \\
\hline COPD & $78(3.7)$ \\
\hline CHF & $76(3.6)$ \\
\hline GI bleed & $75(3.6)$ \\
\hline Cellulitis & $73(3.5)$ \\
\hline Headache & $69(3.3)$ \\
\hline Alcohol abuse & $69(3.3)$ \\
\hline Weakness & $66(3.2)$ \\
\hline Others Diagnosis & $325(15.6)$ \\
\hline \multicolumn{2}{|l|}{ Outside Information Types } \\
\hline Outside medical records & $1635(77.9)$ \\
\hline Outside laboratory results & $389(18.5)$ \\
\hline Outside imaging results & $382(18.2)$ \\
\hline Outside history and physical test results & $255(12.2)$ \\
\hline Outside notes & $206(9.8)$ \\
\hline Outside consultation & $173(8.2)$ \\
\hline Outside discharge summary & $164(7.8)$ \\
\hline Outside EKG results & $153(7.3)$ \\
\hline Outside surgery or procedure notes & $151(7.2)$ \\
\hline
\end{tabular}

Table 3 Common health problems seen and outside information types requested during hospitalizations in the Internal Medicine Department of the Tampa General Hospital.

COPD: congestive obstructive pulmonary disease, CHF: congestive heart failure, EKG: electrocardiogram, GI: gastrointestinal 


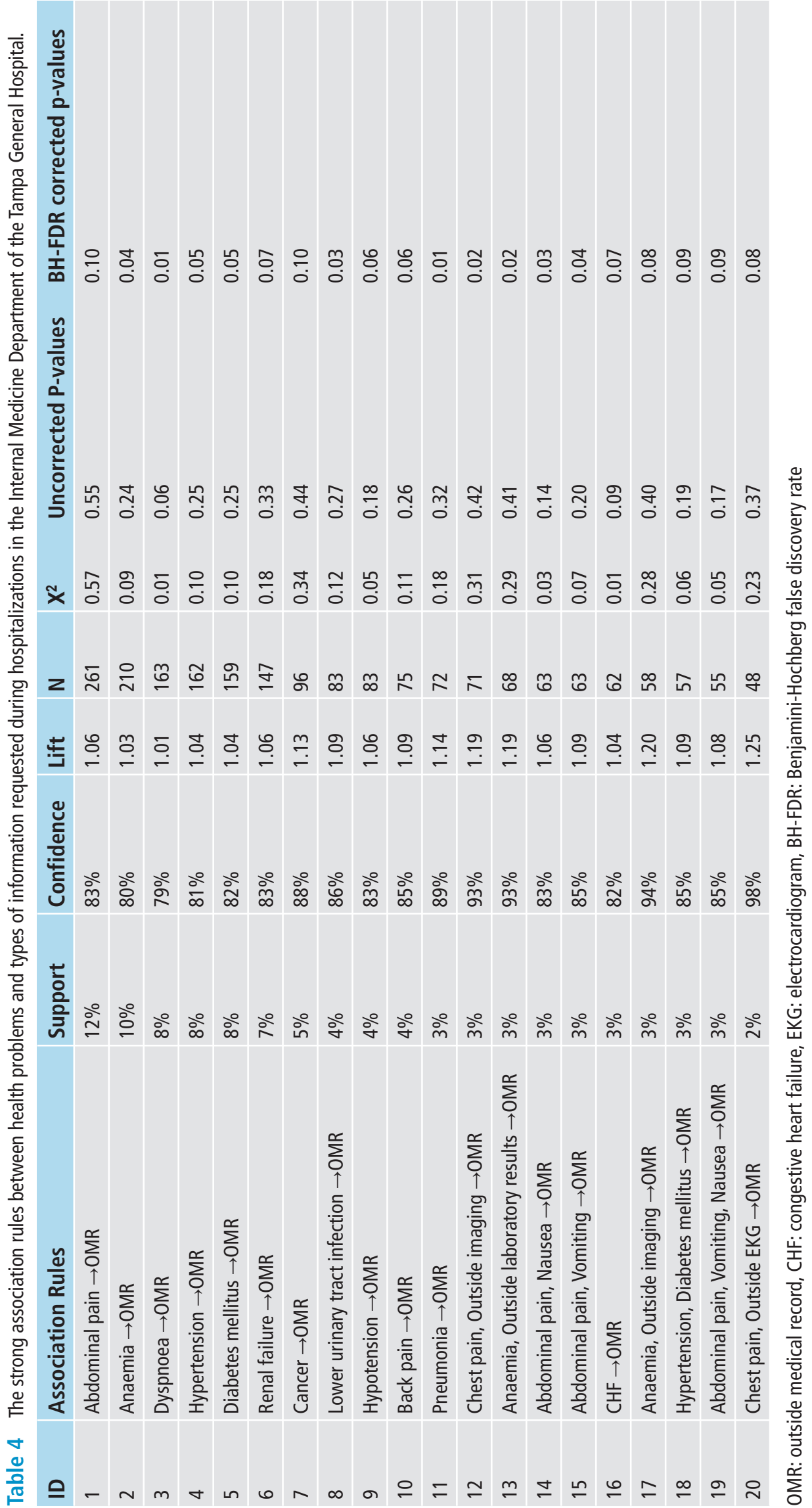




\section{References}

1. Ward BW, Schiller JS, Goodman RA. Multiple chronic conditions among US adults: a 2012 update. Prev Chronic Dis 2014; 11: E62.

2. Anderson G. Chronic Conditions: Making the Case for Ongoing Care. Princeton, NJ: Robert Wood Johnson Foundation 2010.

3. Lucas DJ, Ejaz A, Haut ER, Spolverato G, Haider AH, Pawlik TM. Interhospital transfer and adverse outcomes after general surgery: implications for pay for performance. J Am Coll Surg 2014; 218(3): 393-400.

4. Kho AN, Lemmon L, Commiskey M, Wilson SJ, McDonald CJ. Use of a Regional Health Information Exchange to Detect Crossover of Patients with MRSA between Urban Hospitals. J Am Med Informatics Assoc 2008; 15(2): 212-216.

5. Bailey JE, Wan JY, Mabry LM, Landy SH, Pope RA, Waters TM, Frisse ME. Does health information exchange reduce unnecessary neuroimaging and improve quality of headache care in the emergency department? J Gen Intern Med 2013; 28(2): 176-183.

6. Bailey JE, Pope RA, Elliott EC, Wan JY, Waters TM, Frisse ME. Health Information Exchange Reduces Repeated Diagnostic Imaging for Back Pain. Ann Emerg Med 2013; 62(1): 16-24.

7. Unertl KM, Johnson KB, Lorenzi NM. Health information exchange technology on the front lines of healthcare: workflow factors and patterns of use. J Am Med Inform Assoc 2012; 19(3): 392-400.

8. Carr CM, Krywko DM, Moore HE, Saef SH. The Impact of a Health Information Exchange on the Management of Patients in an Urban Academic Emergency Department: An Observational Study and Cost Analysis. Ann Emerg Med 2012; 60(4): S15.

9. Vest JR, Miller TR. The association between health information exchange and measures of patient satisfaction. Appl Clin Inform 2011; 2(4): 447-459.

10.Solberg D, Roberts J. "Pipe dream" HIE proves challenging. A community hospital network, concerned that each clinic's needs could not be entirely met, decided on a standard EHR platform and a shared community network. Health Manag Technol 2009; 30(7): 22-23, 30.

11. Furukawa MF, King J, Patel V, Hsiao C-J, Adler-Milstein J, Jha AK. Despite Substantial Progress In EHR Adoption, Health Information Exchange And Patient Engagement Remain Low In Office Settings. Health Aff (Millwood) 2014; 33(9): 1672-1679.

12. Adler-Milstein J, Jha AK. Health information exchange among U.S. hospitals: Who's in, who's out, and why? Healthc (Amst) 2014; 2(1): 26-32.

13. Rudin RS, Motala A, Goldzweig CL, Shekelle PG. Usage and Effect of Health Information Exchange: A Systematic Review. Ann Intern Med 2014; 161(11): 803-812.

14. Richardson JE, Abramson EL, Kaushal R. The value of health information exchange. J Healthc Leadersh 2012; 4: 17-23.

15. Vest JR, Jasperson 'S, Zhao H, Gamm LD, Ohsfeldt RL. Use of a health information exchange system in the emergency care of children. BMC Med Inform Decis Mak 2011; 11: 78.

16. Hincapie AL, Warholak TL, Murcko AC, Slack M, Malone DC. Physicians' opinions of a health information exchange. J Am Med Inform Assoc 2011; 18(1): 60-65.

17. Kassirer JP. Doctor discontent. N Engl J Med 1998; 339(21): 1543-1545.

18. Fischman J. Who will take care of you? US News World Rep 2005; 138(4): 44-46.

19. Mechanic D. Physician discontent: challenges and opportunities. JAMA 2003; 290(7): 941-946.

20.Morrison I, Smith R. Hamster health care. BMJ 2000; 321(7276): 1541-1542.

21. Morrison I. The Future of Physicians' Time. Ann Intern Med 2000; 132(1): 80-84.

22. Trude S. So much to do, so little time: physician capacity constraints, 1997-2001. Track Rep 2003; 8: 1-4.

23. Sicotte C, Paré G. Success in health information exchange projects: Solving the implementation puzzle. Soc Sci Med 2010; 70: 1159-1165.

24. Karsh B-T. Clinical Practice Improvement and Redesign: How Change in Workflow Can Be Supported by Clinical Decision Support. Rockville, Maryland: Agency for Healthcare Research and Quality 2009: 1-34.

25. Stead WW, Lin HS. Computational Technology for Effective Health Care: Immediate Steps and Strategic Directions. Washington (DC): National Academies Press (US) 2009: 121.

26. Kripalani S, LeFevre F, Phillips CO, Williams MV, Basaviah P, Baker DW. Deficits in communication and information transfer between hospital-based and primary care physicians: implications for patient safety and continuity of care. JAMA 2007; 297(8): 831-841.

27. Stross JK, Harlan WR. The dissemination of new medical information. JAMA 1979; 241(24): 2622-2624.

28. Strasser TC. The information needs of practicing physicians in Northeastern New York State. J Med Libr Assoc 2012; 100(4 Suppl): G.

29. Curry L, Putnam RW. Continuing medical education in Maritime Canada: The methods physicians use, would prefer and find most effective. Can Med Assoc J 1981; 124(5): 563-566. 
30. Cohen SJ, Weinberger M, Mazzuca S, McDonald CJ. Perceived influence of different information sources on the decision-making of internal medicine house staff and faculty. Soc Sci Med 1982; 16(14): 1361-1364.

31. Northup DE, Moore-West M, Skipper B, Teaf SR. Characteristics of Clinical Information-Searching: Investigation Using Critical Incident Technique. J Med Educ 1983; 58(11): 873-881.

32. Kochen M, Cohen L, Wulff Y. Information systems and clinical research by residents in internal medicine. Methods Inf Med 1985; 24(2): 85-90.

33. Stinson ER, Mueller DA. Survey of health professionals' information habits and needs. Conducted through personal interviews. JAMA 1980; 243(2): 140-143.

34. Christensen DB, Wertheimer AI. Sources of information and influence on new drug prescribing among physicians in an HMO. Soc Sci Med 1979; 13A(3): 313-322.

35. Covell DG, Uman GC, Manning PR. Information needs in office practice: are they being met? Ann Intern Med 1985; 103(4): 596-599.

36. Rudin R, Volk L, Simon S, Bates D. What Affects Clinicians' Usage of Health Information Exchange? Appl Clin Inform 2011; 2(3): 250-262.

37. Abdullah U, Ahmad J, Ahmed A. Analysis of effectiveness of apriori algorithm in medical billing data mining. Proceedings - 4th IEEE International Conference on Emerging Technologies 2008, ICET 2008: $327-331$.

38. Sharma N, Om H. Extracting Significant Patterns for Oral Cancer Detection Using Apriori Algorithm. Intell Inf Manag 2014; 6: 30-37.

39. Ilayaraja M, Meyyappan T. Mining medical data to identify frequent diseases using Apriori algorithm. Proceedings of the 2013 International Conference on Pattern Recognition, Informatics and Mobile Engineering, PRIME 2013: 194-199.

40. Vest JR, Zhao H, Jasperson J, Jaspserson J, Gamm LD, Ohsfeldt RL. Factors motivating and affecting health information exchange usage. J Am Med Inform Assoc 2011; 18(2): 143-149.

41. Agrawal R, Imieliński T, Swami A. Mining association rules between sets of items in large databases. ACM SIGMOD Rec 1993; 22: 207-216.

42. Hahsler M, Buchta BG, Hornik K. Arules: Mining Association Rules and Frequent item sets. 2015. https://cran.r-project.org/web/packages/arules/index.html.

43. Alvarez SA. Chi-squared computation for association rules: preliminary results. Boston, MA: Boston College 2003.

44.Benjamini Y, Hochberg Y. Controlling the false discovery rate: a practical and powerful approach to multiple testing. J R Stat Soc Ser B 1995; 57(1): 289-300.

45.Smith K. Effective communication with primary care providers. Pediatr Clin North Am 2014; 61(4): 671-679.

46. Koopman RJ, Steege LMB, Moore JL, Clarke MA, Canfield SM, Kim MS, Belden JL. Physician Information Needs and Electronic Health Records (EHRs): Time to Reengineer the Clinic Note. J Am Board Fam Med 2015; 28(3): 316-323.

47.Landrigan CP, Conway PH, Edwards S, Srivastava R. Pediatric hospitalists: a systematic review of the literature. Pediatrics 2006; 117(5): 1736-1744.

48. Stewart BA, Fernandes S, Rodriguez-Huertas E, Landzberg M. A preliminary look at duplicate testing associated with lack of electronic health record interoperability for transferred patients. J Am Med Inform Assoc 2010 17(3): 341-344

49. Pantilat SZ, Lindenauer PK, Katz PP, Wachter RM. Primary care physician attitudes regarding communication with hospitalists. Am J Med 2001; 111(9B): 15S-20S.

50. Schabetsberger T, Ammenwerth E, Andreatta S, Gratl G, Haux R, Lechleitner G, Schindelwig K, Stark C, Vogl R, Wilhelmy I, Wozak F. From a paper-based transmission of discharge summaries to electronic communication in health care regions. Int J Med Inform 2006; 75(3-4): 209-215.

51.Laye PG. Tying up loose ends. Thermochim Acta 1997; 300(1): 237-245.

52.El-Kareh R, Roy C, Brodsky G, Perencevich M, Poon EG. Incidence and predictors of microbiology results returning postdischarge and requiring follow-up. J Hosp Med 2011; 6(5): 291-296.

53. Roy CL, Poon EC, Karson AS, Ladak-Merchant Z, Johnson RE, Maviglia SM, Gandhi TK. Patient safety concerns arising from test results that return after hospital discharge. Ann Intern Med 2005; 143(2): 121-128.

54. Walz SE, Smith M, Cox E, Sattin J, Kind AJH. Pending laboratory tests and the hospital discharge summary in patients discharged to sub-acute care. J Gen Intern Med 2011; 26(4): 393-398.

55. Van Walraven C, Laupacis A, Seth R, Wells G. Dictated versus database-generated discharge summaries: A randomized clinical trial. CMAJ 1999; 160(3): 319-326.

56. Teach RL, Shortliffe EH. An analysis of physician attitudes regarding computer-based clinical consultation systems. Comput Biomed Res 1981; 14(6): 542-558. 
57. Singer J, Sacks HS, Lucente F, Chalmers TC. Physician attitudes toward applications of computer data base systems. JAMA 1983; 249(12): 1610-1614.

58. Campion TR, Edwards AM, Johnson SB, Kaushal R. Health information exchange system usage patterns in three communities: practice sites, users, patients, and data. Int J Med Inform2013; 82(9): 810-820.

59. Johnson KB, Unertl KM, Chen Q, Lorenzi NM, Nian H, Bailey J, Frisse M. Health information exchange usage in emergency departments and clinics: the who, what, and why. J Am Med Inform Assoc 2011; 18(5): 690-697.

60. Ozkaynak M, Brennan PF. Revisiting sociotechnical systems in a case of unreported use of health information exchange system in three hospital emergency departments. J Eval Clin Pract 2013; 19(2): 370-373. 